\title{
Variation in Arsenic Metabolism in Schistosomiasis Associated Bladder Pathology in a Rural Community, Eggua, Ogun State, Nigeria
}

\section{${ }^{1} \mathrm{ADEBOYE}, \mathrm{AT} ;{ }^{2} \mathrm{AWOBODE}, \mathrm{HO} ;{ }^{1,3} \mathrm{ADEBAYO}, \mathrm{AS} ;{ }^{4} \mathrm{DJOUAKA}, \mathrm{JR}$; ${ }^{5}$ ISOKPEHI; RD; "6ANUMUDU, CI}

\author{
${ }^{1}$ Cell Biology and Genetics Unit, Department of Zoology, University of Ibadan, Ibadan, Nigeria \\ ${ }^{2}$ Parasitology Unit, Department of Zoology, University of Ibadan, Ibadan, Nigeria \\ ${ }^{3}$ Department of Twin Research and Genetic Epidemiology, King's College, London, United Kingdom \\ ${ }^{4}$ Agro-Eco Health Laboratory, IITA-Benin, Nigeria \\ ${ }^{5}$ School of Science, Mathematics and Engineering, Bethune-Cookman University, Florida, USA \\ ${ }^{* 6}$ Cellular Parasitology Programme, Department of Zoology, University of Ibadan, Ibadan, Nigeria
}

*Corresponding Author Email: cianumudu@yahoo.com; Tel: +2348023590478

\begin{abstract}
Exposure to toxic inorganic Arsenic (iAs) in areas endemic for urogenital schistosomiasis may confer increased risk for bladder cancer. The severity of the adverse effects of iAs however depends on its metabolism, which is highly variable among individuals. Genetic polymorphism in Arsenic (+3) Methyl Transferase enzyme, accounts significantly for these variations. To investigate the relationship of AS3MT gene polymorphisms and Arsenic metabolism to schistosomiasis and/or associated bladder pathology, 119 individuals from Eggua in southwest Nigeria were recruited for this study. Screening for schistosomiasis and bladder pathology was done by microscopy and ultrasonography respectively. Wagtech Digital Arsenator was used to assess total urinary arsenic concentrations and thus determine the level of arsenic exposure. The single nucleotide polymorphism AS3MT/Met287Thr T>C (rs11191439) was genotyped using Alelle-Specific PCR. Of the participants who tested positive for schistosomiasis, 33.3\% exhibited bladder pathology. Total urinary arsenic concentration in $80 \%$ of the participants was above the WHO limit of $0.05 \mathrm{mg} / \mathrm{L}$. The Met287Thr allelic distribution conformed to the Hardy-Weinberg equilibrium $\left(\mathrm{X}^{2}=0.161, \mathrm{P}>0.05\right)$. Observed allelic frequencies were 0.96 and 0.04 for wild-type $\mathrm{T}$ and mutant $\mathrm{C}$ alleles respectively. There was no significant relationship between AS3MT SNP, arsenic concentrations and schistosomiasis associated bladder pathology. In conclusion, the community is highly exposed to arsenic, although with a possible genetic advantage of increased AS3MT catalytic activity. However, we see the need for urgent intervention as inter-individual differences in arsenic metabolism may influence the bladder pathology status of individuals in the community. And although urogenital schistosomiasis is waning in Eggua, it is not known what synergy the infection and high arsenic exposure may wield on bladder pathology.
\end{abstract}

\section{DOI:https://dx.doi.org/10.4314/jasem.v25i9.16}

Copyright: Copyright $\odot 2021$ Adeboye et al. This is an open access article distributed under the Creative Commons Attribution License (CCL), which permits unrestricted use, distribution, and reproduction in any medium, provided the original work is properly cited.

Dates: Received: 09 May 2021; Revised: 12 August 2021; Accepted: 12 September 2021

Keywords: Urogenital schistosomiasis, Arsenic metabolism, polymorphism, Bladder pathology, Bladder cancer

Schistosoma haematobium is a biological carcinogen implicated in a large number of bladder cancer cases in Africa and Middle East (Mbanefo et al., 2018). About 120 million cases of $S$. haematobium infection causing urogenital schistosomiasis (UGS) have been reported in sub-Saharan Africa, with high infection prevalence localised in rural communities. It is believed that in concert with other risk factors associated with bladder cancer, UGS may become the most important factor for bladder cancer globally (Honeycutt et al., 2014). Hence, increased risk for bladder cancer associated with $S$. haematobium infection could come from the progression of schistosomiasis associated bladder pathologies or the occurrence of more severe pathologies (Honeycutt et al., 2014 and Onile et al., 2016). On the other hand, chronic exposure to inorganic (iAs) through contaminated drinking water has been identified as one of the important risk factors associated with bladder cancer in areas not endemic to schistosomiasis but where untreated underground water is being consumed (D'lpplotti et al., 2015). Approximately, 140 million people in 50 countries (including some in Africa), consume inorganic Arsenic (iAS) in contaminated drinking water at concentrations that exceed the World Health Organization's recommended standard of $0.01 \mathrm{mg} / \mathrm{L}$ (WHO, 2018). We recently reported a concordance in exposure to iAS and UGS infection in Eggua, Nigeria (Bakare et al., 2018). Fernandez et al. (2020) in a recent study, also reported that arsenic exposed communities present with more aggressive bladder cancer phenotypes than non-exposed communities. The severity of the adverse effects of AS on human health depends on its metabolism. Successful metabolism of arsenic involves complete and efficient methylation from iAs to di-methylarsenic (DMA) (Recio-Vega et $a l .$, 2016). There is, however, great variation in iAs metabolite concentrations and profile between species, populations, and individuals. Polymorphisms 
in arsenic metabolising genes, such as AS3MT which codes for the enzyme mediating reduction and biomethylation of toxic inorganic arsenic to its less toxic organic forms accounts significantly for these variations (Antonelli et al., 2014). Of great relevance is the Met287Thr (rs11191439) polymorphism which has been found to critically influence arsenic metabolism by elevating MMA urine concentration in studies conducted in different countries (Reviewed by Agusa et al., 2015); the frequency of this polymorphism ranges from 5-10\%. Higher urinary MMA fractions and lower DMA are markers of incomplete and inefficient arsenic metabolism. In humans, a higher percentage of urinary MMA has been associated with increased susceptibility to hypertension, peripheral vascular disease, diabetes, increased risk of lung cancer, skin cancer, and bladder cancer (Antonelli et al., 2014 and Janasik et al., 2014). Therefore, the aim of this study was to investigate the association between $A S 3 M T$ gene polymorphisms and total urinary arsenic concentrations in relation to schistosomiasis associated bladder pathology in the study population.

\section{MATERIALS AND METHODS}

Study area and participants: The study area Eggua, Yewa North Local Government Area of Ogun State is located on a land size of about $200,213.5 \mathrm{~km}^{2}$ with a population size of 255,700 (Adekeye et al., 2021). The inhabitants who are Yoruba speaking people are mainly farmers and traders although some are fishermen. The area is surrounded by many rivers including River Iju, River Yewa, River Balogun, and River Idi which serve as the major sources of water within the community. They are thus foci of transmission of schistosomiasis and possible source of arsenic exposure. Previous studies in this community have reported a high prevalence of schistosomiasis associated bladder pathology as well as high arsenic exposure (Onile et al., 2016 and Bakare et al., 2018). Ethical approval was obtained from the Ogun State Ministry of Health. Participants recruited were adults between 18-75 years old, and voluntarily gave an informed consent before inclusion in the study.

Questionnaires: Information on socio-demographic factors (age, sex, religion and occupation), lifestyle factors (smoker, non-smoker, secondary smoker) occupational history, water source types (well, bore hole, river), and previous health history were obtained through questionnaires.

Collection of Samples: Water samples (1 Litre) were collected from River Iju, River Yewa and River Idi in clean plastic bottles and transported to the laboratory where the level and concentration of Arsenic was determined. Peripheral blood samples $(2 \mathrm{ml})$ were also collected from participants by venepuncture into sterile EDTA bottles. Midstream urine samples were collected between $10 \mathrm{am}$ to $2 \mathrm{pm}$ by participants in $25 \mathrm{ml}$ capacity, universal containers. All samples were labelled appropriately and transported on ice to the laboratory for storage at $-20^{\circ} \mathrm{C}$.

Ultrasonography for bladder pathology: Participants were examined by ultrasound on the field by a radiologist. Parameters used to draw inferences on the overall bladder condition of individual participants were as described by Onile et al., (2016). Bladder thickness of more than $5.0 \mathrm{~cm}$ was reported as pathology and individuals with more than three types of bladder pathology were considered as having severe pathology.

Determination of S. haematobium infection: Urine examination for $S$. haematobium eggs was carried out using standard centrifugation method. Terminally spined $S$. haematobium eggs were identified microscopically. The number of eggs per $10 \mathrm{ml}$ of urine determined the intensity of infection; $0 \mathrm{egg} / 10$ $\mathrm{mL}$ of urine was reported as negative, 1-49 eggs/10 $\mathrm{mL}$ of urine as light intensity of infection and $\geq 50$ eggs $/ 10 \mathrm{~mL}$ of urine as heavy intensity of infection (Onile et al., 2016).

Determination of arsenic levels in water and urine: Total arsenic concentrations in water and urine samples were detected with the Wagtech (WAGWE1050) Digital Arsenator (Palintest Limited, United Kingdom), following the manufacturer's instructions. Detection of total arsenic concentrations in urine samples was however optimized based on the procedure described by Khan and Ahmad (2015). This involved dilution of urine samples by a factor of 4 , addition of $1 \mathrm{ml}$ potassium iodide ascorbic solution, a double concentration of sulphamic acid and longer reaction time (50 minutes). Forty urine samples were analysed for total arsenic concentrations; these were then categorized into four groups according to their schistosomiasis and bladder pathology status. Individuals negative for schistosomiasis and with no bladder pathology served as the control. Group 1 comprised of individuals positive for schistosomiasis only, Group 2 comprised of individuals positive for schistosomiasis infection and having bladder pathology and Group 3 comprised of individuals with bladder pathology only.

AS3MT/Met287Thr SNP Genotyping: Genotyping of AS3MT gene polymorphisms was conducted using the two step AS-PCR method (Darawi et al., 2013). A set of common primers (AS3MT-Fc and AS3MT-Rc) was first used to amplify and flank the region containing the polymorphism. In the second round two sets of primers, the common primers (AS3MT-Fc and AS3MT-Rc), a forward T-allele specific primer (AS3MT-Fas-T) and a reverse C-allele specific primer (AS3MT- Ras-C) were used. The sequences of the common primers were retrieved from previous 
studies (Farhid et al., 2017 and Recio-vega et al., 2016) where the polymorphism had been genotyped using PCR-RFLP. The allele- specific primers were however designed de novo using the NCBI primer blast tool after retrieving the gene sequence from NCBI Gen- bank (Table 1).

Table 1: Sequence of primers and expected fragment lengths

\begin{tabular}{lll}
\hline PRIMER & SEQUENCE (5'-3') & FRAGMENT LENGTH (BP) \\
NAME & GTGCTGGAGATGAACCGTGAA & 232 \\
\hline AS3MT-Fc & GCAAGGGCAAGAGCAGAAAGA & “ \\
AS3MT-Rc & AGGACATGAAAAAGAACTAAT & 99 \\
AS3MT-Fas-T & ATGTAAAATTGGCATCAAACG & 175 \\
AS3MT-Ras-C & ATA & The specific nucleotides at the 3'-end of primers were underlined
\end{tabular}

First round PCR: Amplification was done in a 25ul reaction mixture comprising $12.5 \mathrm{ul}$ of $1 \mathrm{x}$ Go Taq Green master mix, $0.15 \mathrm{ul}$ each of forward common (AS3MT-Fc) and reverse common (AS3MT-Rc), 7.2 ul nuclease free water and 5 ul of template DNA with PCR cycling condition of initial denaturation at $95^{\circ} \mathrm{C}$ for 3 mins followed by 30 cycles of denaturation at $94^{\circ} \mathrm{C}$ for $30 \mathrm{~s}$, annealing at $60{ }^{\circ} \mathrm{C}$ for $30 \mathrm{sec}$ and extension at $72^{\circ} \mathrm{C}$ for $1 \mathrm{~min}$ followed by a final extension of $72^{\circ} \mathrm{C}$ for 5 mins. The amplification was confirmed through visualisation on $1.5 \%$ agarose gel stained with ethidium bromide.

Second round PCR: The cocktail mixture of the second round PCR comprised of $12.5 \mathrm{ul} 1 \mathrm{x}$ Go Taq green master mix, $2 \mathrm{ul}$ of first round $\mathrm{PCR}$ product, 0.25 ul each of AS3MT-Fc, AS3MT-Rc, AS3MT- Fas- T and AS3MT- Ras- C, 9.5 ul nuclease free water.

The PCR cycling conditions were an initial denaturation of $95^{\circ} \mathrm{C}$ for 3 mins followed by 30 cycles of denaturation at $94^{\circ} \mathrm{C}$ for $30 \mathrm{secs}$, annealing at $50^{\circ} \mathrm{C}$ for $30 \mathrm{~s}$, and extension at $72^{\circ} \mathrm{C}$ for $30 \mathrm{~s}$ followed by a final extension at $72^{\circ} \mathrm{C}$ for 5mins. After the amplification, electrophoresis was performed at 100 $\mathrm{V}$ for $50 \mathrm{~min}$ in $1 \mathrm{X}$ TBE buffer on $1.5 \%$ agarose gel stained with ethidium bromide. The amplified PCR products were visualized with Quantum CX5 Edge gel imaging/ documentation system (Fisher Biotec, Australia).

Statistical analysis: Descriptive statistics including percentage values was used to analyse data obtained from the questionnaire. Statistical package for social sciences (SPSS) for windows software packages was used to analyse all data. Chi-square test was used to determine significant relationships between variables and in testing deviation from Hardy Weinberg equilibrium in haplotypes.

Logistic regression analysis was used to determine the relative impact of SNP haplotypes, urinary Arsenic concentration and schistosomiasis on associated bladder pathologies. A confidence interval of $95 \%$, and $\mathrm{p}$ values of less than 0.05 was considered to be significant except while testing for Hardy Weinberg equilibrium where p-value $>0.05$ was considered significant.

\section{RESULTS AND DISCUSSION}

A total of 151 participants were recruited for the study; however, 32 participants were excluded because of missing information and/or samples vital to the study. Thus, the analysis was based on 119 participants who provided all the necessary information and samples. Of the 119 participants, 86 $(72.3 \%)$ were female and 33(27.7\%) were male (Table 2).

Prevalence and intensity of $S$. haematobium in the study population: The prevalence of $S$. haematobium infection was 5\%; 6/119 participants (3males and 3 females) were positive for $S$. haematobium. Participants in the age group 10-30 had the highest prevalence with $4(19.05 \%)$ of them being infected. In addition, only $2(5.3 \%)$ of the participants who were above 50 years old were infected.

Table 2: Demographic and Descriptive statistics of the stud
population


There was a significant difference $(\mathrm{P}=0.01$; $\mathrm{X}^{2}=9.137$ ) in $S$. haematobium infection among the different age groups but not between gender $(\mathrm{P}=$ $\left.0.211 ; X^{2}=1.564\right)$. The overall mean intensity of schistosomiasis in the study population was 39.2 eggs/10ml of urine. A mean intensity of 65 eggs/10ml of urine and $13.33 \mathrm{eggs} / 10 \mathrm{ml}$ of urine were recorded for infected female and male participants respectively. However, from the questionnaire data, 13/119 participants $(10.9 \%)$ indicated that they had been previously diagnosed of $S$. haematobium infection. Of these 13 participants, 2(15\%) had a reoccurring infection with a mean intensity of $15 \mathrm{eggs} / 10 \mathrm{ml}$ of urine.

Bladder pathology in the study population: All 119 participants were examined for bladder and urinary tract pathology; $36(30.25 \%)$ were found to have one type of pathology or the other. The common bladder pathology types observed were bladder wall thickness $(100 \%)$, bladder mass $(5.5 \%)$ and hydronephrosis $(8.5 \%)$. Bladder pathologies in the population were more common among males than females with a prevalence of $48.5 \%$ and $23.3 \%$ respectively. Statistical analysis showed that there was a significant relationship between gender and bladder pathology
$\left(\mathrm{P}=0.007, \mathrm{X}^{2}=7.194\right)$. However, there was no significant difference between bladder pathology in the age groups $\left(\mathrm{P}<0.05: \mathrm{P}=0.670, \mathrm{X}^{2}=0.802\right)$.

Bladder pathology and schistosomiasis: Out of 36 participants positively diagnosed for bladder pathology, only two individuals tested positive for a current $S$. haematobium infection while five had a previous history of schistosomiasis.

Arsenic levels in water and urine samples: Only River Iju, out of the four water bodies, was positive for arsenic contamination at a concentration of $0.8 \mathrm{mg} / \mathrm{L}$, which is above the WHO standard limit of $0.01 \mathrm{mg} / \mathrm{L}$ for arsenic in drinking water. Total urinary arsenic concentration in this study ranged from 0 to $>0.8 \mathrm{mg} / \mathrm{L}$ with a mean of $0.22 \mathrm{mg} / \mathrm{L}$. Out of the 40 urine samples analysed, 32(80\%) had detectable arsenic concentration (ranging from 0.08 to 0.80 ) above the WHO standard limit of $0.05 \mathrm{mg} / \mathrm{L}$ for arsenic in urine, three $(7.5 \%)$ were below the WHO standard limit and 5(12.5\%) had no detectable level of arsenic in their urine. With respect to age groups there was no statistical difference in the mean total arsenic concentration estimated by one-way ANOVA (F $(2,35)=0.192, \mathrm{p}=0.826)$.

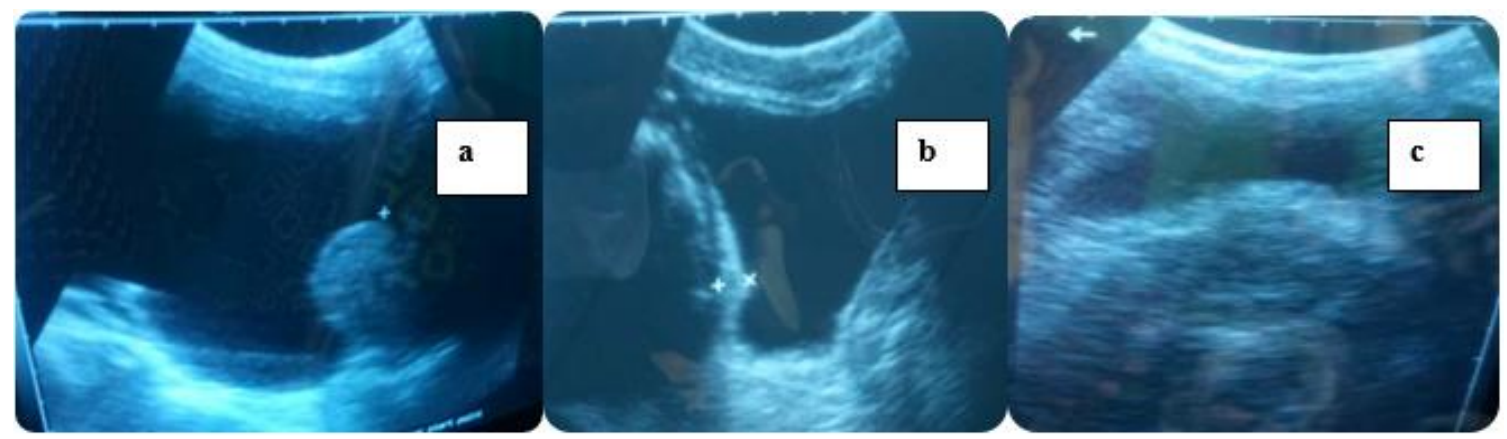

Fig 1: Ultrasound imaging of some bladder pathologies among participants (a). Bladder mass lesion in the lateral wall of the bladder (b) Thick bladder wall (c) Co-incidence of hydronephrosis and enlarged prostate.

Total arsenic concentrations across the groups examined is shown in Figure 2. The control group had the highest mean concentration of total arsenic $(0.41 \mathrm{mg} / \mathrm{L})$, followed by the pathology group with a mean concentration of $0.18 \mathrm{mg} / \mathrm{L}$. There was a significant difference in arsenic concentrations between groups as determined by one-way ANOVA $(\mathrm{F}(3,36)=4.434, \mathrm{p}=0.009)$.

Frequency of Met287Thr/AS3MT (rs11191439) SNP in the study population: The genotyping data for AS3MT/M287T polymorphisms are shown in Figures $4 \mathrm{a}$ and $4 \mathrm{~b}$. In this study there was no homozygous mutant. The frequency of homozygous TT genotype and heterozygous TC genotype was $91.7 \%$ and $8.3 \%$ respectively. The observed allelic frequencies, i.e. the probability of each allele ( $\mathrm{T}$ and $\mathrm{C}$ ) occurring in the population, were calculated to be 0.96 and 0.04 for $\mathrm{T}$ and C alleles respectively. The SNP rs11191439 conformed to Hardy-Weinberg equilibrium where $\mathrm{X}^{2}=0.161$ with $\mathrm{P}$-value $>0.05$.

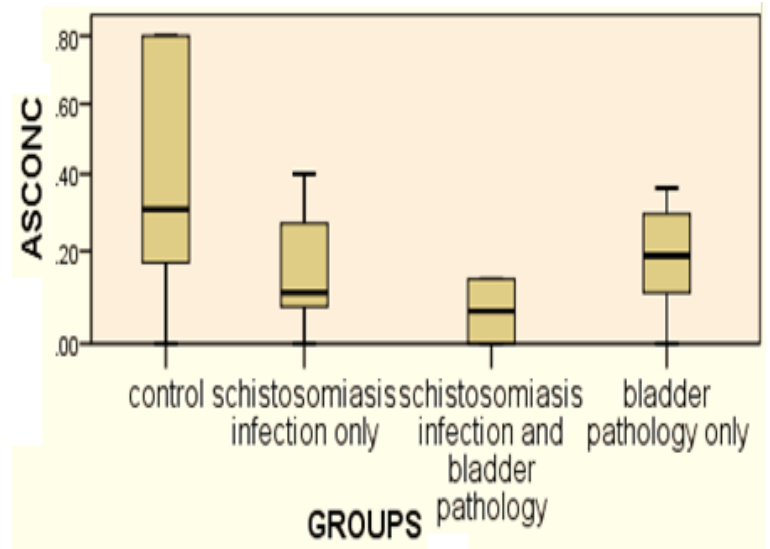

Fig 2: Total urinary arsenic concentrations among participants grouped according to their schistosomiasis and bladder pathology 


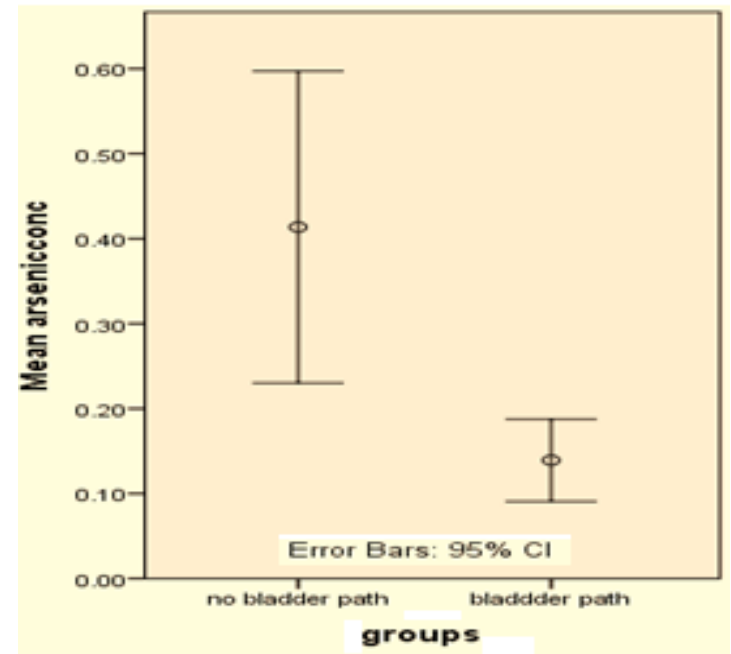

Fig 3: Mean concentration of urinary arsenic was higher in bladder pathology cases than in the no bladder pathology group

MET287THR/AS3MT polymorphism and total arsenic concentrations: All the individuals whose urine arsenic levels were within the WHO limit had the wild type homozygous TT genotype and there was no recorded case of either TC or CC genotype, while in the 32 individuals with arsenic levels above the WHO limit, 30(93.8\%) had the TT genotype and 2(6.2\%) had the heterozygous TC genotype. There was no significant difference between AS3MT SNPs and total arsenic concentrations $\left(\mathrm{X}^{2}=0.127, \mathrm{p}<0.05, \mathrm{p}=0.722\right)$ as shown in Table 3.

MET287THR/AS3MT polymorphism, Bladder pathology and schistosomiasis: Of the 30 cases with bladder pathology that were genotyped, 27 had TT genotype and three were of TC genotype. While in the normal bladder cases, 28 had TT genotype and two had TC genotype. There was no significant difference between polymorphism and bladder pathology $\left(\mathrm{X}^{2}=0.281, \mathrm{P}<0.05, \mathrm{P}=0.640\right)$. In two cases of joint schistosomiasis infection and bladder pathology incidence, $1(50 \%)$ had TT genotype and $1(50 \%)$ had TC genotype.

\section{MET287THR/AS3MT}

polymorphism,

schistosomiasis, total arsenic concentrations and Bladder pathology: The logistic regression model was statistically significant $\left(\mathrm{X}^{2}(3)=15.530, \mathrm{p}=0.01\right)$. The model explained $63.7 \%$ (Nagelkerke $\mathrm{R}^{2}$ ) of the variance in bladder pathology and correctly classified $83.3 \%$ of cases. The model indicated that high total arsenic concentration was associated with a reduction in the likelihood of exhibiting bladder pathology, with an estimated odds ratio of 0.00008 and $95 \%$ CI of odds ratio at $0.0000-0.179(\mathrm{P}=0.022)$ as shown in Table 4. Hence, increase in total urinary arsenic concentration confers approximately $99.9 \%$ reduction in chances of having bladder pathology. The individual contribution of other factors i.e polymorphism and schistosomiasis was statistically insignificant $(\mathrm{p}=0.29$ and $\mathrm{p}=0.116$ respectively).
There was, however, no significant relationship between AS3MT SNP, arsenic metabolism, and schistosomiasis associated bladder pathology $\left(\mathrm{R}^{2}=\right.$ 0.159, $\mathrm{P}=0.094)$.

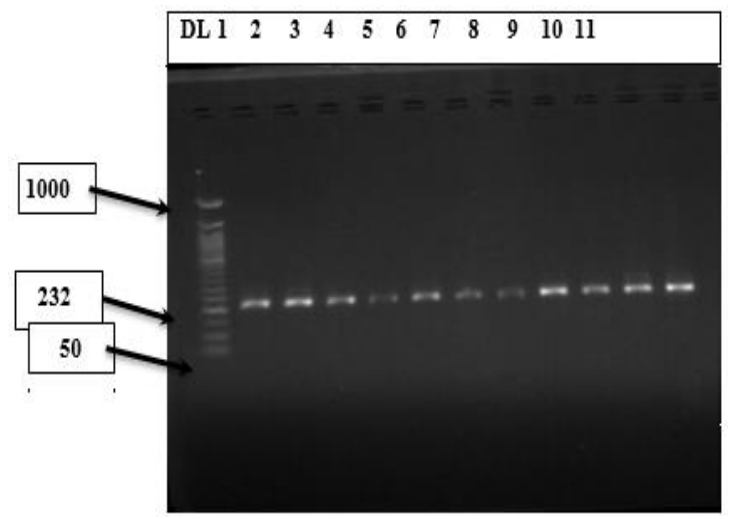

Fig 4a: First round PCR products of amplified AS3MTregion with the expected fragment length of $232 \mathrm{bp}$. DL- $1 \mathrm{~kb}$ DNA ladder.

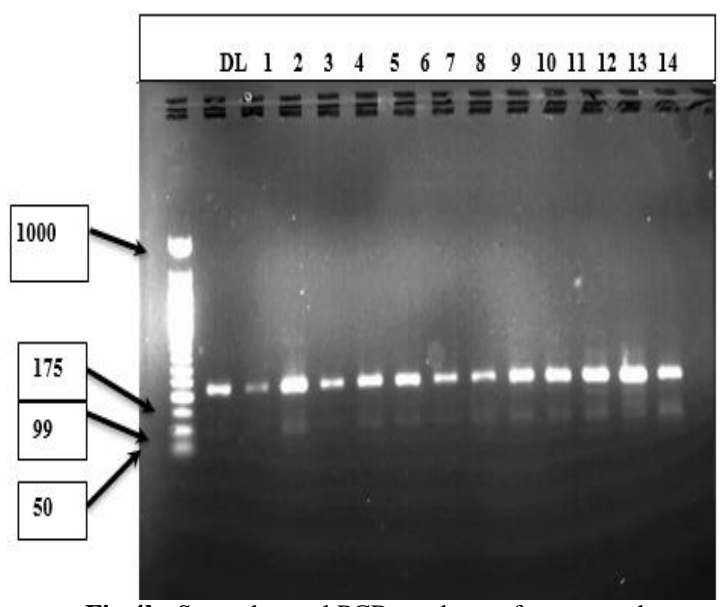

Fig 4b: Second round PCR products of genotyped MET287THR/AS3MT polymorphism. Wild type homozygous TT showed bands at 232 and 99 bp while mutant heterozygous carriers TC showed bands at 233, 175 and 99 bp (lane 11 and 12). DL- $1 \mathrm{~kb}$ DNA ladder.

Table 3: Urinary arsenic concentrations associated with MET287THR/AS3MT polymorphism

\begin{tabular}{|c|c|c|c|c|}
\hline \multirow{2}{*}{$\begin{array}{l}X^{2}=0.127 \\
P=0.722\end{array}$} & & \multicolumn{2}{|c|}{$\begin{array}{l}\text { Arsenic } \\
\text { concentration }\end{array}$} & \multirow[t]{2}{*}{$\begin{array}{l}\text { Total } \\
\text { N }(\%)\end{array}$} \\
\hline & & $\begin{array}{l}<0.05 \\
\mathrm{mg} / \mathbf{l} \\
\mathrm{N}(\%)\end{array}$ & $\begin{array}{l}>0.05 \mathrm{~m} \\
\mathrm{~g} / \mathrm{l} \\
\mathrm{N}(\%)\end{array}$ & \\
\hline & $\begin{array}{l}\mathrm{T} \\
\mathrm{T}\end{array}$ & $2(6.3)$ & $30(93.7)$ & $32(8.6)$ \\
\hline Polymorphism & $\begin{array}{l}\mathrm{T} \\
\mathrm{C}\end{array}$ & $1(33.3)$ & $2(66.7)$ & $3(91.4)$ \\
\hline Total & & $3(91.4)$ & $32(8.6)$ & 35 \\
\hline
\end{tabular}

Schistosomiasis and Bladder pathology in the study population: Urinary schistosomiasis is highly endemic in Nigeria and tropical Africa. Some studies in Nigeria have reported very high prevalence levels of between $30-50 \%$ amongst adults and $40-60 \%$ in children (Abdulkadir et al., 2017). However, in recent years there has been a gradual decline in schistosomiasis prevalence in adults in the study population. Adekeye et al., (2021), reported an 
average prevalence of $26.5 \%$ in Yewa North, which was indeed lower than the Nigerian estimated mean prevalence of $34.7 \%$, reported by Abdulkadir et al. (2017). Notably, in this present study, a significant reduction in urinary schistosomiasis prevalence was observed in adults, with prevalence and mean intensity being $5 \%$ and 39.2 eggs/10ml of urine respectively. This decline may be attributed to the Mass administration of deworming treatment regimens (including Praziquantel) to school children in the area by the State Ministry of Health in the last 6years, and through research activity in this community. It could also be due to some control and preventive interventions such as the provision of a borehole for the community, which in theory should minimise the people's contact with S. haematobium cercariae. Perhaps the famously high limestone deposits in this area extends to the rivers as well as climate change, might have also affected the ecology of the snail host at the contact sites. The prevalence of bladder pathology in this study was $30.3 \%$, with a 2.76 increased risk in males, similar to $33.9 \%$ prevalence previously reported by Onile et al. (2016) in this study area. Adeoye and Ipeajeda (1994) reported that older infections with schistosomiasis are associated with bladder calcification and fibrosis which lead to smaller numbers of eggs or no visible eggs excreted in the urine. This might explain why $S$. haematobium ova were not detected in the bladder pathology cases with past history of schistosomiasis infection presented in this study. Therefore, these individuals might not be totally free of schistosomiasis infection; they may be false negatives, or rather advanced cases showing more chronic symptoms as retention of eggs in the bladder also increases the degree of bladder morbidity. These data again underscore the need for a good reliable molecular diagnostic for schistosomiasis.

Table 4: Model coefficient from logistic regression analysis showing the risk factors and their predictive strength on bladder pathology

\begin{tabular}{lllllllll}
\hline & B & S.E. & Wald & df & Sig. & Exp(B) & \multicolumn{2}{c}{ 95\% C. I } \\
\cline { 4 - 8 } Factors & & & & & & & Lower & Upper \\
\hline AS conc (mg/l) & -11.685 & 5.083 & 5.285 & 1 & .022 & .00008 & .000 & .179 \\
Polymorphism (1) & 3.477 & 3.324 & 1.094 & 1 & $.296^{* *}$ & 32.362 & .048 & 21854.122 \\
Schisto (1) & -2.566 & 1.632 & 2.471 & 1 & $.116^{* *}$ & .077 & .003 & 1.884 \\
Constant & 3.272 & 1.411 & 5.379 & 1 & .020 & 26.364 & & \\
\hline \multicolumn{8}{c}{ Values insignificant at $p<0.05$}
\end{tabular}

Urinary arsenic in the study population: Mean total urinary arsenic concentration in this study was $0.22 \mathrm{mg} / \mathrm{l}$, and $80 \%$ of the samples had arsenic concentrations exceeding the $0.05 \mathrm{mg} / \mathrm{L}$ WHO standard limit for arsenic. The mean total urinary arsenic concentration observed in this study is higher than $0.07 \mathrm{mg} / \mathrm{L}$ observed by Farzan et al. (2015) in a cross-sectional study of U.S adults but lower than $1.16 \mathrm{mg} / \mathrm{L}$ observed by Bakare et al. (2018) in the study area. According to Chanda et al., (2011), high levels of arsenic in the urine could be indicative of reduced body burden of the metalloid. Thus, it would be almost safe to conclude that although these individuals are highly exposed to the metalloid, their metabolism is also capable of rapidly expelling it in urine, decreasing the risk for arsenic-associated clinical symptoms. Hence, a low overall excretion rate and arsenic retention in tissues would increase the risk for carcinogenesis (Recio-vega et al., 2020). Indeed, our data on mean urine arsenic concentrations in bladder pathology cases compared to those with no bladder pathology (Fig 3), as well as logistic regression analyses seem to buttress this conclusion.

Arsenic in the rivers: Contaminated underground drinking water is considered as the main source of inorganic arsenic exposure. Majority of individuals in the study $(58 \%)$ population rely on underground water sources for drinking water. However, only one of the rivers showed arsenic contamination, with levels lower than our previous observations for the same river (Bakare et al., 2018). This might indicate a need to investigate other non-water sources of arsenic for the high community exposure observed; these sources could be food and food crops, especially rice, since in this area rice farming is becoming a huge cottage industry. Contaminated groundwater can increase the arsenic content of various parts of rice plants (Agusa et al., 2019). We may also consider a longitudinal measurement of this metalloid in the water body as its concentration may be dependent on other factors which come into play at different times in the year. Fernandez et al. (2020) also reports that individuals may be exposed to arsenic through inhalation in communities where mining activities occur.

AS3MT Met287Thr polymorphism: The TC/CC genotype frequency observed in this study was lower than $12 \%$ observed in Bangladeshi men and women, but higher than $4.2 \%$ reported in Vietnamese men and women (Agusa et al., 2009). The frequency of C allele (0.04) in this study is close to 0.01 reported in Japan and Turks as opposed to 0.11 reported in Iran (Fujihara et al., 2009 and Farhid et al., 2017). Farhid et al. (2017) reported that a lower frequency (0.000.041) of $A S 3 M T \mathrm{C}$ allele may confer higher enzyme activity and may have a genetic advantage for individuals who are at high risk of arsenic exposure. Thus, we hypothesized that the high urinary concentrations of arsenic found in this population could be as a result of increased enzyme activity and high tolerance due to very low occurrence of the $\mathrm{C}$ allele. Due to the low numbers of cases of confirmed schistosomiasis, confirmed associated bladder 
pathology and the low prevalence of the polymorphism in the study, it was difficult to assign increased risk of bladder pathology to the MET287THR/AS3MT polymorphism and we were unable to better explore the susceptibility or risk of individuals in the sample population to severe schistosomiasis associated bladder pathologies.

Conclusion: Arsenic exposure is high in this area. However, the low frequency of the AS3MT gene polymorphism in individuals indicates increased arsenic metabolism. Urinary arsenic concentrations may influence bladder pathology in the population, in that the higher the concentration of arsenic expelled in urine, the lower the risk for bladder pathology. Also, our nine years long search-and-treat surveys may have contributed to the waning prevalence of schistosomiasis in Eggua, but not to its high concordance with bladder pathology. There is a need to replicate this study on a larger scale to validate our findings.

Acknowledgements: We acknowledge Murielle Farelle Soglo for her technical assistance in the laboratory assessment of Arsenic in IITA-Benin. CIA acknowledges WHO/RCS/KM grant B40394.

\section{REFERENCES}

Abdulkadir, A; Ahmed, M; Abubakar, BM; Suleiman, IE; Yusuf, I; Imam, IM; Sule, AA; Tela, UM; Dogo, HM; Yakasai, AM; Musa, BM (2017). Prevalence of urinary schistosomiasis in Nigeria, 1994-2015: Systematic review and metaanalysis. Afr. J. Urol. 23(4).

Adekeye, TA; Onile, OS; Adebayo, AS; Osundiran, AJ; Anumudu, CI; Awobode, HO (2021). Spatial distribution and ecology of soil parasites and Schistosoma haematobium infection in Eggua, Nigeria. Environ. Adv. 5, 100080. doi.org/10.1016/j.envadv.2021.100080

Adeoye, GO; Ipeajeda, M (1994). Schistosoma haematobium infection among school children in Owena Barracks, Akure, Nigeria. Nig. J Parasitol. 17: 51-61

Agusa, T; Iwata, H; Fujihara, J; Kunito, T; Takeshita, H; Minh, TB; Tanabe, S. (2009). Genetic polymorphisms in AS3MT and arsenic metabolism in residents of the Red River Delta, Vietnam. Tox. App. Pharmacol. 236(2):131-141.

Agusa, T; Kunito, T; Fujihara, J; Takeshita, H; Takahashi, S; Tanabe, S; Iwata, H (2015). Relationship between arsenic (+ 3 oxidation state) methyltransferase genetic polymorphisms and methylation capacity of inorganic arsenic. Jap J. Hyg. 70(3): 186-196.
Antonelli, R; Shao, K; Thomas, DJ; Sams, R 2 ${ }^{\text {nd; }}$ Cowden, J. (2014). AS3MT, GSTO, and PNP polymorphisms: Impact on arsenic methylation and implications for disease susceptibility. Environ. Res. 132: 156-167.

Bakare, SO; Adebayo, AS; Awobode, HO; Onile, OS; Agunloye, AM; Isokpehi, RD; Anumudu, CI (2018). Arsenicosis in bladder pathology and schistosomiasis in Eggua, Nigeria. Trans. Roy. Soc. Trop. Med. Hyg. 112(5): 230-237.

Chanda, S; Gangul, B; Dasgupta, U (2011). GST polymorphic status modifying the arsenic induced clinical manifestation in people of the southern part of West Bengal, India. J. Toxicol. Environ. Health Sci. 3:171-175.

Darawi, MN; Ai-Vyrn C; Ramasamy K; Hua, PPJ; Pin, TM; Kamaruzzaman, SB; Majeed, ABA. (2013). Allele-specific polymerase chain reaction for the detection of Alzheimer's disease-related single nucleotide polymorphisms. BMC Med Genet. 14(1): 27.

D'Ippoliti, D; Antelli, E; DeSario, M, Scortichini, M., Davoli, M. and Michelozzi, P., (2015). Arsenic in drinking water and mortality for cancer and chronic diseases in central Italy,1990-2010. PLoS One. 10(9): e0138182. doi.org/10.1371/journal.pone.0138182

Farhid, F; Nadali, F; Chahardouli, B; Mohammadi, S; Rostami, S; Alimoghaddam, K; Ghavamzadeh, A (2017). Frequency of M287T/AS3MT Single Nucleotide Polymorphism in an Iranian Population. Int J Hematol Oncol Stem Cell Res. 11(1):19-23

Farzan, SF; Li, Z; Korrick, SA; Spiegelman, D; Enelow, R; Nadeau, K; Baker, E; Karagas, MR (2015). Infant infections and respiratory symptoms in relation to in utero arsenic exposure in a US cohort. Environ health Perspect. 124 (6):840-847.

Fernández, MI; Valdebenito, P; Delgado, I; Segebre, J; Chaparro, E; Fuentealba, D; Castillo, M; Vial, C; Barroso, J.P; Ziegler, A; Bustamante, A (2020). Impact of arsenic exposure on clinicopathological characteristics of bladder cancer: A comparative study between patients from an arsenic-exposed region and non-exposed reference sites. Urol. Oncol. 38(2):40.e1-40.e7

Fujihara, J; Fujii, Y; Agusa, T; Kunito, T; Yasuda, T; Moritani, T; Takeshita, H (2009). Ethnic differences in five intronic polymorphisms associated with arsenic metabolism within human arsenic (+ 3 oxidation state) methyltransferase 
(AS3MT) gene. J Toxicol Appl Pharmacol. 234(1): 41-46.

Honeycutt, J; Hammam, O; Fu, CL; Hsieh, MH (2014). Controversies and challenges in research on urogenital schistosomiasis-associated bladder cancer. Trends in parasitol, 30(7), 324-332.

Khan, MH; Ahmad, SK (2015). Field detection method of Arsenic in Urine. J of Prev. Soc. Med. 34 (2): 10-16

Mbanefo, EC; Hsieh, MH (2018). Defining the Pathways of Urogenital SchistosomiasisAssociated Urothelial Carcinogenesis through Transgenic and Bladder Wall Egg Injection Models. Methods in Mol Biol 1655:67-76

Onile, OS; Awobode, HO; Oladele, VS; Agunloye, AM; Anumudu, CI (2016). Detection of urinary tract pathology in some Schistosoma haematobium infected Nigerian Adults. J Trop Med. Article ID 5405207: 1-5.
Recio-Vega, R; González-Cortes, T; OlivasCalderón, E; Clark Lantz, R; Jay Gandolfi, A; Michel-Ramirez, G (2016). Association between polymorphisms in arsenic metabolism genes and urinary arsenic methylation profiles in girls and boys chronically exposed to arsenic. Environ. Mol. Mutagen. 57(7): 516 -525.

Recio-Vega, R; Hernandez-Gonzalez, S; MichelRamirez, G; Olivas-Calderón, E; Lantz, RC; Gandolfi, AJ; Amistadi, MK (2021). Association between the polymorphism of three genes involved in the methylation and efflux of arsenic (As3MT, MRP1, and P-gp) with lung cancer in a Mexican cohort. J. App. Toxicol. 41(9): 13571366.

WHO (2018). Exposure to Arsenic: A Major Public Health Concern. WHO Switzerland 